\title{
QUENCHING OF EXCITED ALKALI ATOMS AND RELATED EFFECTS IN FLAMES: PART I. THEORETICAL ANALYSIS
}

\author{
H. P. Hooymayers and C. Th. J. Alkemade \\ Physical Laboratory, University of Utrecht. Netherlands
}

(Received 13 December 1965)

\begin{abstract}
An a.c. photoelectric detection device has been used to determine the yield factor $p$ of resonance fluorescence of the $\mathrm{Na}(5890 / 96) \cdot \AA$ and $\mathrm{K}(7665 / 99) \AA$ and $(4044 / 47) \AA$ resonance doublets in atmospheric flames as a function of the composition and temperature of the flame. From these measurements the value (and temperature dependence) of the specific effective quenching cross-sections of the considered lines for $\mathrm{N}_{2}, \mathrm{CO}_{2}$, $\mathrm{CO}, \mathrm{O}_{2}, \mathrm{H}_{2}, \mathrm{Ar}$ and $\mathrm{H}_{2} \mathrm{O}$ could be derived. The experimental procedure and the results obtained will be reported and discussed in Part II of this paper. In Part I a theoretical analysis is given of the effect of radiative nonequilibrium on the occupation of the excited state, as a function of the $p$-value, the metal concentration in the flame, and the distance from the flame surface. In particular, an approximate expression for the population (or source) function in the high density case is derived by an iteration procedure for assumed rectangular, Doppler and Lorentzian spectral line profiles respectively. It is assumed that the source function is independent of frequency and that the excited level combines with the ground state only. In the analysis a homogeneous and isothermal slab of flame gases between two plane parallel boundaries is considered. Diffusion effects of the atomic metal vapour at the flame border are considered semi-quantitatively. Also the effect of radiative nonequilibrium on the intensity of the outgoing radiation. the line-reversal temperature, the shape of the curve of growth, and the intensity ratio of the doublet lines is analysed. Finally, the expected influence of quenching collisions on the broadening of spectral lines in flames is calculated.
\end{abstract}

\section{INTRODUCTION}

THE YIELD factor $p$ of (resonance) fluorescence is defined according to the Voigt--Volmer formula as the fractional probability that an excited atom loses its energy by radiation of a photon. Assuming a two-level atomic model and denoting the radiative transition probability per sec by $A$, we have $p=A /\left(k_{-1}+A\right)$, where $k_{-1}$ describes the probability per sec that the excited atom loses its energy in a quenching collision with a flame particle. If $A$ is known, the value of $k_{-1}$ can be found by measuring $p$. Knowledge of the quenching probability per sec provides information on the non-adiabatic interaction between the electronic state of the atom and the quenching particle. By applying detailed balancing, the efficiency of excitation by collisions of first kind can be derived from the quenching effects measured. A detailed knowledge of excitation and quenching mechanisms in flames is desired when deviations from thermodynamic equilibrium in flames are discussed. These deviations are of interest in spectroscopic flame research as well as in practical flame photometry. We may mention, for example, the investigations of chemiluminescent reactions in which flame radicals play a dominant part. ${ }^{(1)}$ Chemiluminescence has also become recently a practical tool in the flame photometric analysis of elements with high excitation levels, such as $\mathrm{Ni}$ and $\mathrm{Sn}^{(2)}$ Furthermore, the recent application of flame fluorescence in practical flame photometry may profit by any extension of our knowledge about fluorescence yield factors. ${ }^{(3)}$ 
The major problem involved in the study of (de-)excitation mechanisms is the question which form of energy the electronic excitation energy is converted into or supplied from. It has been known for a long time, from fluorescence measurements in metal vapour bulbs with foreign gases added, that (noble gas) atoms have much lower (de-)excitation elliciences than molecules. Conversion of electronic excitation energy into translational energy, and vice versa, is obviously an improbable process. Therefore it is believed that (de-)excitation of metal atoms in flames proceeds primarily by conversion of excitation energy into internal molecular (rotational and/or vibrational) energy. Conversion into vibrational energy seems to be favoured, since here no restrictions resulting from conservation of linear and angular momentum are involved. Strong evidence for this conclusion may be derived from shock-tube experiments. These showed that the apparent $\mathrm{Na}$-excitation temperature follows closely the relaxation of the vibrational temperature of the molecular compound present in the shocked gas. ${ }^{(4-7)}$ Also recent experiments on the excitation of the electronic state of sodium by vibrationally excited nitrogen molecules which were produced in the after-glow of a microwave discharge in a flowing $\mathrm{N}_{2}$ stream, point to the same conclusion. ${ }^{(8)} \mathrm{A}$ possible (small) resonance defect of the order $k T$ ( $k=$ Boltzmann factor; $T=$ flame-temperature), between the electronic excitation energy and the energy of the nearest vibrational level may be readily compensated by additional exchange of rotational and translational energy. In Part II of this paper, further detailed evidence for the important contribution of molecular vibrational energy to the excitation of alkali atoms will be presented which is based on the observation of certain resonance effects obtained with diatomic molecules. It may be noted that, in turn, conversion of vibrational energy into translational energy may be favoured by ladder effects: the higher vibrational levels are populated step by step by transitions from the lower-lying levels. The energy required for each step is of the order $k T$ in flames and may be supplied readily from the available translational energy of the particles involved in the exciting collision. Hence the $\mathrm{Na}$ line-reversal temperature measured in flames is indirectly linked up with the "true" translational temperature of the flame gas through the energy distribution of the molecular vibrational levels. It should be noted, however, that relaxation effects in this energy distribution, as found in shock-tubes, will be hardly manifest in flames at $1 \mathrm{~atm}$, since the flow-velocity of the flame gases is comparatively low (about $0 \cdot 1-1 \mathrm{~cm} /$ millisec). Moreover the relaxation time is markedly reduced by the presence of water vapour in flames. Therefore it may be assumed that the "vibrational temperature" of the flame molecules is identical with the true translational flame temperature.

We first summarize some of the relevant literature data. For a survey of earlier and more recent literature we may refer to MiTCHELL and ZEMANSKY ${ }^{(9)}$ and CALLEAR ${ }^{(43)}$ respectively. BEUTLER and JOSEPHY ${ }^{(10)}$ showed by experiments on the sensitized fluorescence of sodium vapour that the efficiency of quenching collisions increased markedly when only a small part of the available energy is converted into kinetic energy of the colliding species. This explains why molecules with many closely-spaced internal energylevels are usually much more effective as quenchers than noble gas atoms.

MANNKOPfF, (11) VON HAMOS, ${ }^{(12)}$ NORRISH $^{(13)}$ and others have measured the dependence of quenching of fluorescence radiation in metal vapour bulbs on pressure and on the kind of foreign gas molecules, at temperatures considerably lower than flame temperatures. The main difficulty in these experiments is the dependence of the spectral line profile of the fluorescent atoms on the pressure of the quenching gas. It is not possible 
to escape this dependence completely, because the quenching collisions do increase the transition probability of the excited Na-atoms.

Demtröder, ${ }^{(14)}$ Hulpke, Paul and Paul ${ }^{(15)}$ and others irradiated sodium vapour in a vessel with light that had been modulated at high frequencies. They measured the influence of quenching collisions on the life-time of excited sodium atoms in the $3 P$-state and then derived quenching cross-sections.

TSUCHIYA, ${ }^{(7)}$ GAYDON, ${ }^{(16)}$ HuRlE $^{(16)}$ and others have investigated and discussed the energy transfer between $\mathrm{Na}$-atoms and various shock-heated diatomic molecules by measuring line-reversal temperatures.

KARL and POLANYI ${ }^{(17)}$ have investigated the infrared emission of carbon monoxide while quenching electronically excited mercury atoms, and found that the electronic energy of the mercury atom was transferred to vibrational energy of carbon monoxide by quenching collisions.

Dickens, LinNETT and SOVERS ${ }^{(18)}$ have tried to calculate quantum-mechanically the cross-section for energy transfer of $\mathrm{Na}$ - and $\mathrm{Hg}$-atoms in collision with diatomic molecules as a function of the resonance defect $\Delta E$ and the relative velocity $v_{r}$. Here $\Delta E$ is the energy difference between the atomic excitation level and the nearest vibrational level of the quenching molecules. The approximations and simplifications applied by these authors turned out to be too crude, however, since their calculated cross-sections for sodium differed from the measured values by many orders of magnitude. For the quenching of excited mercury atoms by $\mathrm{N}_{2}$-molecules, they predicted a pronounced resonance effect which is in qualitative agreement with our experience. (See Part II.)

The experimental literature data on quenching cross-sections for the alkali resonance lines still lack overall agreement and completeness, whereas theory cannot give reliable estimates for these values. In particular, no data are available at all for the quenching effect of $\mathrm{H}_{2} \mathrm{O}$ molecules, which are a major constituent in flame gases. The variation of quenching cross-section over a large range of temperatures or relative velocities has not yet been established, so that conversion of data obtained at lower temperatures to flame conditions is hardly possible. On the other hand, the investigations in progress in our laboratory concerning the systematic errors in line-reversal temperature measurements (SNELLEMAN $^{(19)}$ ) the absolute rate of chemiluminescent reactions (ZEEGERS, ALKEMADE ${ }^{(20)}$ ), and the interpretation of curves-of-growth in terms of spectral line-broadening (HoLLANDER $^{(21,42)}$ ) are strongly in need of supplementary data on fluorescence yield factors for the alkali resonance lines under flame conditions at $1 \mathrm{~atm}$. Some preliminary results of our investigation for the $\mathrm{Na}$ D-doublet have been briefly communicated in two previous papers. ${ }^{(1,22)}$

\section{THEORETICAL ANALYSIS}

\section{Influence of radiative non-equilibrium on occupation of excited states}

We first discuss theoretically the influence of radiative non-equilibrium which depends on the fluorescence yield factor $p$, on the occupation of the excited metal state for the extreme cases of low and high metal density, rcspectively. Using a computer, IEARN ${ }^{(23)}$ has already calculated the exact source function (i.e. the ratio of the emission coefficient to the absorption coefficient), and from it the line profiles and radiation intensities for an infinite plane parallel slab of plasma. HEARN's calculations were performed for a number 
of $p$-values $(\geq 0.5)$ and density values in the case of pure Doppler broadened resonance lines only. CUPERMAN, ENGELMANN and OXENIUS ${ }^{(24.25)}$ derived an approximative analyt ical expression for the source function and the total outgoing de-excitation radiation for a spherical plasma, when again only Doppler broadening is taken into account.

In our work we have attempted to derive an analytical expression for these quantities in the low and high-density case, respectively, and for an infinite plane parallel homogeneous slab of flame gases. In our calculations we have not only considered Doppler broadened but also Lorent $z$ broadened lines. because in flames at atmospheric pressure and temperatures of about $2000^{\circ} \mathrm{K}$ the two broadening effects are of comparable magnitude, in contrast to most plasma work. An approximate expression of the source function is derived by applying an iteration procedure. Then an expression for the net flame emission and the deviation of line reversal temperatures from the true flame temperature is derived. This expression holds, to first order, in $p$ in the case of pure Doppler and pure Lorentz broadened line profiles, and to second order in $p$ for a rectangular line profile. (See Notation.)

The occupation of the excited metal state obeys the Boltzmann formula exactly only if the excitation and de-excitation rates are in detailed balance. We then have $N_{1}^{0} / N_{0}=$ $g_{1} / g_{0} \exp (-E / k T)$ where $N_{0}$ and $N_{1}^{0}$ are the number of atoms per $\mathrm{cm}^{3}$ in the ground and excited state, $g_{0}$ and $g_{1}$ are the corresponding statistical weight factors of the state, and $E=h v_{0}$, where $h$ is Planck's constant and $v_{0}$ is the central frequency of the emitted resonance radiation. The application of this formula would require that the flame is placed inside a black-body with the same temperature $T$ as the flame so that the radiation density at each place in the flame corresponds to Planck's radiation law. We shall

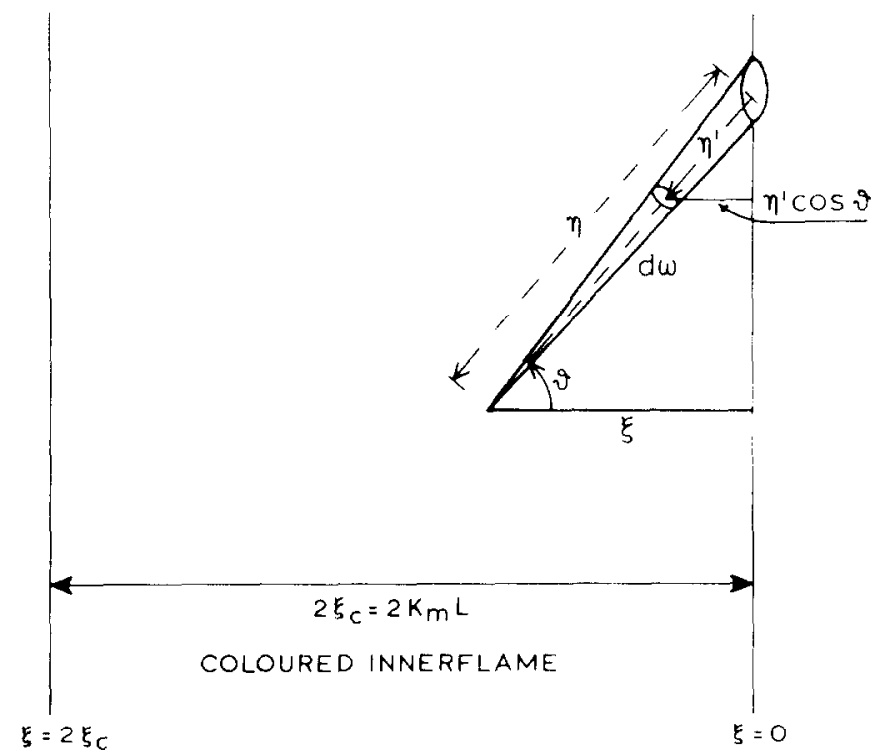

FIC. 1. Cross-section through slab of flame gases with thickness $2 \mathrm{~L}$. perpendicular to flame surface: $\xi=\kappa_{m} x$. where $x=$ distance of considered point to flame surface and $\kappa_{m}=$ absorption coefficient at spectral line center. 
investigate here, to what degree this condition is fulfilled in flames and what deviations from Boltzmann's law are to be expected.

Let us first suppose we are working under conditions such that the radiation density at the frequency of the metal line is virtually zero (i.e. low metal concentration, nonincandescent flame, no radiation influx from external light sources). Excitation of metal atoms then occurs mainly through collisions of the first kind with flame molecules with a rate constant $k_{1}$. In a stationary flame, we have the over-all balance $N_{0} k_{1}=N_{1}\left(k_{-1}+A\right)$, if $k_{-1}$ is the rate constant for the reverse quenching collisions and $A$ is the optical transition probability per sec. $N_{1}$ is the occupational number of the excited state, which will now in general be lower than the thermodynamic equilibrium value $N_{1}^{0}$. The values of $k_{1}$ and $k_{-1}$ are, in general, dependent on the energy distribution of the colliding species. Since, however, the metal-particles are present only as a trace in the flame gases it is not expected that any deviation of the population of the excited state from Boltzmann equilibrium will upset the equilibrium distribution for the flame particles, hence the values of $k_{1}$ and $k_{-1}$ will be the same as in complete thermodynamic equilibrium, so that we have for the actual values $N_{0} k_{1}=N_{1}^{0} k_{-1}$. Combining both expressions for $N_{0} k_{1}$ we find immediately

$$
N_{1}=\frac{k_{-1}}{k_{-1}+A} \cdot N_{1}^{0}=(1-p) N_{1}^{0}
$$

with $p=A /\left(k_{-1}+A\right)=$ yield factor of resonance fluorescence. It should be noted that equation (1) holds uniformly at any place inside the coloured flamepart* (see Fig. 2).

We now consider the other extreme case of high atomic metal density, i.e. high selfabsorption in the flame. Since the collisional excitation is essentially thermal, we may expect now that the radiation density at the line center and in the midplane of the flame slab will approach asymptotically the Planck radiation density at the flame temperature. Consequently, at the mid-plane, $N_{1}$ will there approach its equilibrium value $N_{1}^{0}$. At the very flame border, the radiation density can never exceed half the Planck density, because the influx of radiation from outside the flame is here essentially zero. We must thus expect that $N_{1}$ will vary as a function of distance from the coloured flame border. In the quantitative evaluation of this variation, we shall assume that the ground state concentration $N_{0}$ is constant throughout the coloured flame section (possible effects of outward diffusion of metal atoms will be considered later) and that at any point in the flame $N_{1} \ll N_{0}$ is valid, which holds if the Boltzmann factor $\exp (-E / k T)$ is very small. This condition is obviously fulfilled for optical alkali lines in flames and also permits us to neglect stimulated emission. In this approximation, the absorption coefficient $\kappa_{v}$ at the frequency $v$ is independent of the spatial coordinates in the flame. Since, in the high density case, the maximum value $\kappa_{m}$ of $\kappa_{v}$ at the line center is, by definition much larger than the reciprocal radius of curvature $(L)^{-1}$ of the flame boundary, we may consider instead of the cylindrical flame actually used (cf. Part II), an infinite slab of flame gases with plane parallel boundaries and thickness $2 L$ (see Fig. 1). The distance of a point in the flame to the flame surface, viewed by the observer, is measured by the coordinate $x$, which runs from 0 to $2 L$. Instead of $x$ we shall also use the dimensionless quantity

\footnotetext{
* The flame used in our experiment (cl. Part II of this paper) was composed of a coloured cylindrical inner flame surrounded by a colourless flame mantle of the same gas composition and temperature and equal flow speed. For brevity we will indicate in the following the coloured inner flame by "the flame".
} 

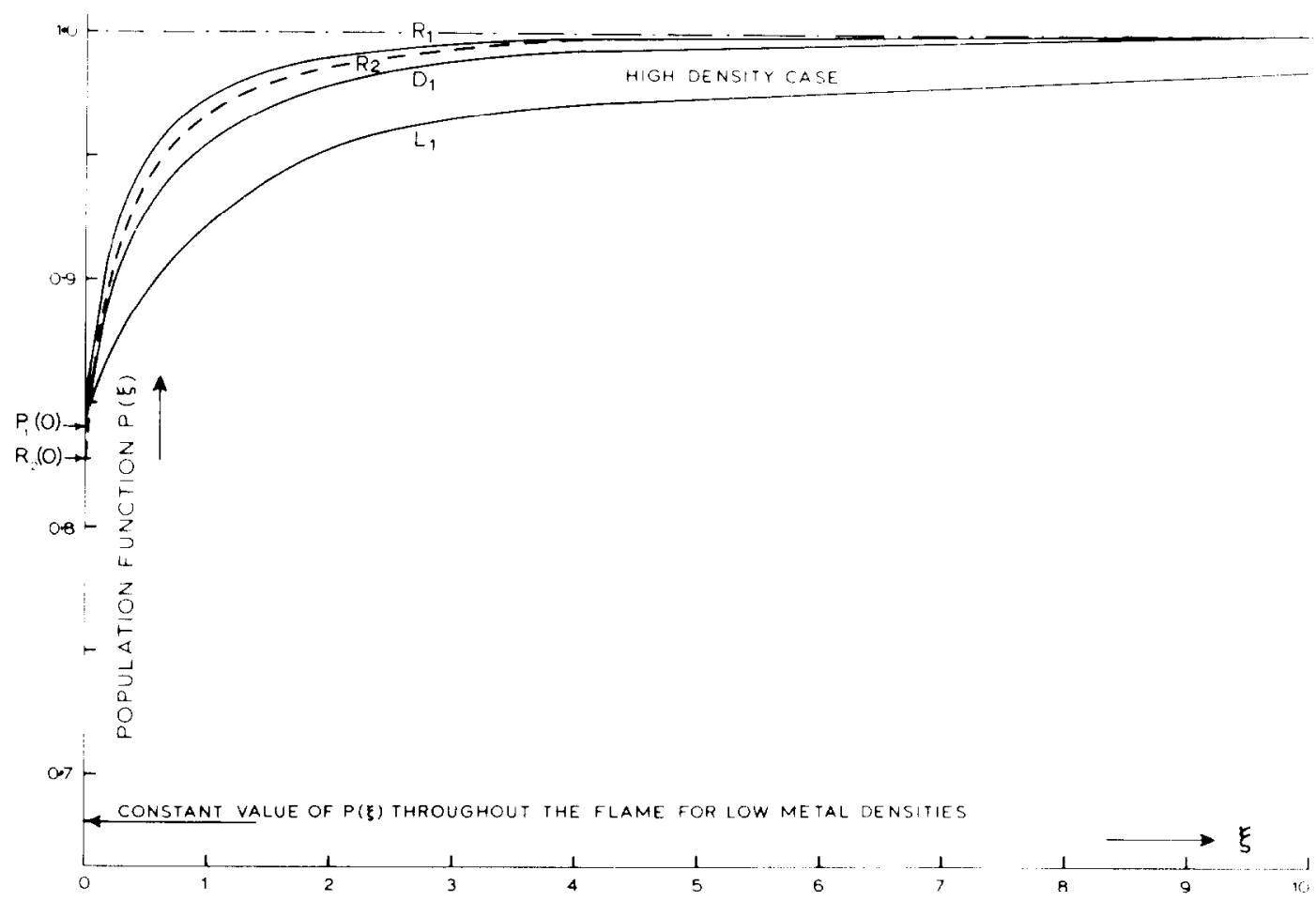

Fig. 2. Population function $P(\xi)$ calculated as a function of dimensionless distance $\xi$ to the flame surface in the low and high density case, respectively. for rectangular $\left(R_{1}\right)$. Doppler $\left(D_{1}\right)$ and Lorentz $\left(\mathrm{L}_{1}\right)$ line profiles in first order approximation of the yield factor $p$ of resonance fluorescence. for $p=0 \cdot 32$. The dashed curve $R_{2}$ represents the second order approximation of the population function calculated for a rectangular line profile in the case of high atomic densities for the same $p$ value

$\xi \equiv x \kappa_{m}$. In the high-density case (because of symmetry), the occupational number $N_{1}$ of the excited state is a function of $\xi$ only. This function $N_{1}(\xi)$, moreover, will be symmetric with respect to the central plane at $\xi=\xi_{c}$. Instead of this function we prefer using the normalized population function $P(\xi)=N_{1}(\xi) / N_{1}^{0}$. The function $P(\xi)$ is identical to the "fractional source function" (i.e. the source function as a fraction of the black-body source function) which is usually introduced in the literature from a different point of view. ${ }^{(26-28)}$ Following Hearn, ${ }^{(29)}$ DOBrowOLnY, ${ }^{(30)}$ ENGELmanN $^{(30)}$ and others, we also assume that the absorption and emission coefficients have the same dependence on frequency. Moreover, we suppose that the distance travelled by an excited atom before it is de-excited is always (much) less than $\kappa_{m}^{-1}$.

Whereas for low densities $P(\xi)$ is constant and equal to $1-p$ (cf. equation (1)). we have for high densities that $P(\xi)$ decreases gradually from unity at flame center $\left(\xi=\xi_{i}\right)$ to a value between $(1-p)$ and $\left(1-\frac{1}{2} p\right)$ at the flame border $(\xi=0$ and $\xi=2 L$, respectively: see Fig. 2). It should be realized that at the very flame border the radiation density is at most half the Planck density at flame temperature.

The calculation of $P(\xi)$ at a given point in the flame requires knowledge of the radiation density $\rho_{v}$ as a function of frequency $v$ at that point. 
In the steady state the rate of de-excitation balances the rate of excitation, and we have, therefore,

$$
\left(A+k_{-1}\right) N_{1}(\xi)=\int_{0}^{\infty} \rho_{v}(\xi) \cdot\left(c / h v_{0}\right) \kappa_{v} \mathrm{~d} v+k_{1} \cdot N_{0}
$$

where the first term in the right-hand side equals the excitation rate by photon absorption at the place $\xi$. By applying the principle of detailed balancing in the case of complete equilibrium, and by denoting

$$
\delta(\xi)=\int_{0}^{\infty} \rho_{v}(\xi) \cdot \kappa_{v} \mathrm{~d} v / \int_{0}^{\infty} \rho_{b} \kappa_{v} \mathrm{~d} v
$$

(with $\rho_{b}=$ black-body radiation density at temperature $T$ ), we find from equation (2)

$$
\left(A+k_{-1}\right) N_{1}(\xi)=\left\{\delta(\xi) \cdot A+k_{-1}\right\} N_{1}^{0} .
$$

Accordingly we get

$$
P(\xi)=N_{1} / N_{1}^{0}=1-p+\delta(\xi) p
$$

when use is made of the definition of $p$. Obviously $\delta=0$ corresponds to the low-density limit discussed above, whereas $\delta=1$ prevails at the flame center in the high-density limit. In equation (3), $\rho_{v}(\xi)$ is found by integrating the radiation intensity $I_{v}(\xi, \theta)$ that is incident on an infinitesimal element at distance $\xi$ from the flame border, under an angle of incidence $\theta$ (see Fig. 1), over all spatial directions ${ }^{(31)}$

$$
\rho_{v}(\xi)=\frac{1}{c} \int_{\omega} I_{v}(\xi, \theta) \mathrm{d} \omega
$$

Here $c$ is light velocity and $\mathrm{d} \omega$ is an infinitesimal solid angle. $I_{v}$, in turn, depends on the fractional population function $P$ according to (see Fig. 1).

$$
\frac{I_{v}(\xi, \theta)}{I_{b}}=\int_{0}^{r} P\left(r^{\prime} \cos \theta \cdot \kappa_{m}\right) \exp \left[-\left(r-r^{\prime}\right) \kappa_{v}\right] \kappa_{v} \mathrm{~d} r^{\prime},
$$

where $I_{b}$ is the black-body radiation intensity at $v_{0}$ and $r$ measures the distance (in $\mathrm{cm}$ ) from the considered element to the flame border along the direction given by 0 . The exponential factor in the integrand follows directly from Beer's law.

Abbreviating $\tau_{v} \equiv r \kappa_{v}$ and defining $\psi_{v} \equiv \kappa_{v} / \kappa_{m}$ we get

$$
\frac{I_{v}(\xi, \theta)}{I_{b}}=\mathrm{e}^{-\tau_{v}} \int_{0}^{\tau_{v}} P\left(\tau_{v}^{\prime} \cos \theta / \psi_{v}\right) \mathrm{e}^{\tau_{v}} \mathrm{~d} \tau_{v}^{\prime} .
$$

This result, which also follows from an integration of the equation of radiation transfer ${ }^{(23)}$ permits us, in combination with equations (3), (5) and (6), to calculate the population function $P$ in successive orders of approximation by an iteration procedure. In the high-density case $\left(\xi_{c} \gg 1\right)$ we find $P_{1}(\xi)$ in first order approximation with respect to the yield factor $p$ by assuming the function $P$ in the integrand of equation (7) to equal unity. This is tantamount to say that $I_{v}$ can be calculated at any place in the flame by assuming 
that the occupation of the excited level obeys Boltzmann's law throughout the flame. Of course, with this assumption, the contribution of the left-hand hemisphere (see Fig. 1) in equation (6) simply equals $2 \pi I_{b} / c$.

We then find denoting $\eta=\kappa_{m} r^{\prime}$

$$
P_{1}(\xi)=(1-p)+\frac{1}{2} p+(p / 4 \pi) \frac{\left.\int_{0}^{0} \mathrm{~d} v \int_{0}^{\pi / 2} \mathrm{~d} \theta\right) \int_{0}^{2 \pi} \mathrm{d} \varphi \sin \theta \exp \left[-\left(\psi_{v} \xi / \cos \theta\right)\right] \int_{0}^{\xi / \cos \theta} \psi_{v}^{2} \exp \left(\psi_{v} \eta\right) \mathrm{d} \eta}{\int_{0}^{k} \psi_{v} \mathrm{~d} v^{\prime}}
$$

where $\varphi$ is the azimuthal angle. Performing the integration over $\varphi$ and $\eta$, we obtain

$$
P_{1}(\xi)=1-\frac{1}{2} p+\frac{1}{2} p \frac{\int_{0}^{\infty} \mathrm{d} v \int_{0}^{\pi / 2} \mathrm{~d} \theta \psi_{v}\left(1-\exp \left[-\left(\psi_{v} \xi / \cos \theta\right)\right]\right) \sin \theta}{\int_{0}^{\pi} \psi_{v} \mathrm{~d} v}
$$

We note that $P_{1}(0)=1-\frac{1}{2} p$ regardless the spectral line shape (see Fig. 2). The meaning of the integral expression in equation (7) is easily understood, if one realizes that in this order of approximation Kirchhoff's law for equilibrium radiation may be applied and that the factor $\left(1-\exp \left[-\psi_{v} \xi / \cos \theta\right]\right)$ in the integrand represents the absorption factor at frequency $v$ of a column of flame gases with length $\xi / \cos \theta$.

Inserting again $P_{1}(\xi)$ found from equation (9), in equation $(7), P_{2}(\xi)$ can--in principle - be found in second order of approximation of the fluorescence yield factor $p$.

We shall now calculate $P_{1}(\xi)$ for some typically different profiles $\psi_{v}$ of the absorption coefficient. First the more simple, be it less realistic, case of a rectangular line shape is considered, which is determined by $\psi_{v}=1$ for $\left|v-v_{0}\right| \leq \frac{1}{2} \Delta v$, and $\psi_{v}=0$ otherwise. This simple dependence on frequency also permits us to investigate in how far $P_{2}(\zeta)$ deviates from $P_{1}(\zeta)$.

Next we shall treat the cases of pure Lorentz and pure Doppler profiles, given by

$$
\psi_{v}=\frac{1}{1+\left(\frac{2\left(v-v_{0}\right)}{\Delta v_{L}}\right)^{2}} \text { and } \psi_{v}=\exp -\left(\frac{2\left(v-v_{0}\right)}{\Delta v_{\mathrm{D}}}[\ln 2]^{\frac{1}{2}}\right)^{2} \text {, respectively }
$$

where $\Delta v_{L}$ and $\Delta v_{D}$ represent the Lorentz and Doppler breadth at half maximum of the absorption coefficient respectively. For convenience we introduce the dimensionless frequency differences

$$
y=2\left(v-v_{0}\right) / \Delta v_{\mathrm{L}} \text { and } y=\frac{2\left(v-v_{0}\right)}{\Delta v_{\mathrm{D}}}(\ln 2)^{\frac{1}{2}}
$$

in the case of a Lorentz and a Doppler profile, respectively.

Case of rectangular line profile

Inserting the expression for $\psi_{v}$ in equation (9) we obtain after some algebra 


$$
P_{1}(\xi)=1-p / 2+p / 2 \int_{0}^{\pi / 2}[1-\exp (-\xi / \cos \theta)] \sin \theta \mathrm{d} \theta
$$

abbreviating $\cos \theta=u$ we get

$$
1-p / 2 \int_{0}^{1} \mathrm{e}^{-\xi / u} \mathrm{~d} u
$$

which, performing the integration over $u$, changes into

$$
\begin{gathered}
=1-p / 2\left[\mathrm{e}^{-\xi}+\xi E i(-\xi)\right] \text { with } 0 \leq \xi \leq \xi_{c}, \\
\operatorname{Ei}(\xi)=\int_{-\infty}^{\xi} \frac{e^{x}}{x} \mathrm{~d} x
\end{gathered}
$$

represents the exponential integral function. For $\xi=\xi_{c}$ and $\xi=0$ we find the expected values 1 and $1-\frac{1}{2} p$, respectively (cf. above).

In order to calculate $P(\xi)$ in second order of $p$ (i.e. $P_{2}(\xi)$ ) we have to insert $P_{1}(\xi)$ in equation (7) and obtain by using equation (5)

$$
\begin{aligned}
P_{2}(\xi) & =1-p+p / 4 \pi \int_{\omega} \exp \left(-\tau_{v}\right) \int_{0}^{\tau_{v}} P_{1} \exp \left(\tau_{v}^{\prime}\right) \mathrm{d} \tau_{v}^{\prime} \\
& =1-p+p / 4 \pi\left(\frac{F^{-}(\xi)+F^{+}(\xi)}{I_{b}}\right) .
\end{aligned}
$$

The integral has been split up into two contributions $F^{+}$and $F^{-}$, (from the right-hand and left-hand hemisphere, respectively) to the total radiation flux incident on the considered element in the flame. Since the function $P_{1}$ occurring in the above integral deviates from its equilibrium value $P=1$, at least near to the flame boundary, we cannot apply Kirchhoff's law in calculating $F^{-}$and $F^{+}$. Instead we have to subdivide the flame volume comprised in the infinitesimal solid angle $\mathrm{d} \omega$, at direction $\theta$, into subelements which are labelled by their dimensionless distance $\xi=\eta \cos \theta$ to the flame border, measured along a normal direction (see Fig. 1). Then one finds

$$
\frac{F^{+}}{4 \pi I_{b}}=\frac{1}{4 \pi} \int_{0}^{2 \pi} \mathrm{d} \varphi \int_{0}^{\pi / 2} \mathrm{~d} \theta \sin \theta \exp (-\xi / \cos \theta) \int_{0}^{\xi / \cos \theta} P_{1}(\eta \cos \theta) \exp (\eta) \mathrm{d} \eta .
$$

Abbreviating $\cos \theta=s$ and substituting equation (10) for $P_{1}(\eta \cos \theta)$ we obtain after some algebra :

$$
\frac{F^{+}(\xi)}{4 \pi I_{b}}=\frac{1}{2}-\frac{1}{2} \int_{0}^{1} \exp (-\xi / s) \mathrm{d} s-p / 4 \int_{0}^{1} \int_{0}^{1} \frac{u}{u-s}[\exp (-\xi / u)-\exp (-\xi / s)] \mathrm{d} u \mathrm{~d} s .
$$

The radiation flux $F^{-}$incident on the considered element is computed along similar lines as $F^{+}$. If we assume that the flame is optically thick, so that $\frac{2}{3} \xi_{c}$ is appreciably larger than 
unity, we have virtually $F^{+}=2 \pi I_{b}=F^{-}$, for points situated in the interval $\frac{2}{3} \xi_{c} \leq \xi \leq \xi_{c}$. For points with $0 \leq \xi \leq \frac{2}{3} \xi_{c}$ we have now only to consider the contribution to $F^{-}$from subelements in the left-hand hemisphere that have a dimensionless distance less than $\frac{2}{3} \xi_{\mathrm{c}}$ from the point considered. Moreover, the value of the normalized population function at points with $\xi \geq \frac{2}{3} \xi_{c}$ may be put equal to unity, we then find after some algebra

$$
\frac{F^{-}(\xi)}{4 \pi I_{b}}=\frac{1}{2}-p / 4 \int_{0}^{1} \int_{0}^{1} \frac{u}{s+u} \exp (-\xi / u) \mathrm{d} u \mathrm{~d} s .
$$

Combining equation (12) and equation (13) we finally obtain for the population function in second order approximation in $p$

$$
\begin{aligned}
P_{2}(\xi)=1-p / 2 \int_{0}^{1} \exp (-\xi / s) \mathrm{d} s-p^{2} / 4 \int_{0}^{1} \mathrm{~d} u \int_{0}^{1} \\
\times \mathrm{d} s\left\{[\exp (-\xi / u)-\exp (-\xi / s)]-\frac{u}{s+u} \exp (-\xi / u)\right\}
\end{aligned}
$$

As can be expected it follows that $P_{2}(\xi)=1$ for $\frac{2}{3} \xi_{c} \leq \xi \leq \xi_{c}$. For $\xi-0, P_{2}(0)-1-p / 2-$ $p^{2} / 8$ which is independent of metal concentration (i.e. $\kappa_{m}$ ) in the high-density case. For arbitrary $\xi$ the value of $P(\xi)$ increases monotonously with $\xi$ between these extreme values (see Fig. 2).

Case of Lorentz line profile

To calculate $P_{1}(\xi)$ for a Lorentz broadened resonance line we insert

$$
\psi_{v} \mathrm{~d} v=\frac{\Delta v_{\mathbf{L}}}{2} \frac{\mathrm{d} y}{1+y}
$$

in equation (9) and arrive at the following relation if we put $\cos \theta=u$

$$
P_{1}(\xi)=1-p / 2+p / 2 \frac{\int_{0}^{\infty} \mathrm{d} y \int_{0}^{1} \mathrm{~d} u \frac{1}{1+y^{2}}\left[1-\exp \left[-\xi / u\left(1+y^{2}\right)\right]\right]}{\int_{0}^{\infty} \mathrm{d} y\left(1+y^{2}\right)^{-1}} .
$$

After integration over $y$ one finds

$$
P_{1}(\xi)=1-p / 2 \int_{0}^{1} \mathrm{~d} u \exp (-\xi / 2 u) J_{0}(i . \xi / 2 u),
$$

where $J_{0}$ is a Bessel function of the first kind.* This result is obtained by applying

* If $J_{v}(i . \xi / 2 u)$ is replaced by $\exp (-\xi / 2 u)$ we find for $P_{1}(\xi)$ the same expression as is found for a rectangular line profile. 
$2 / \pi \int_{0}^{\infty} \mathrm{d} y \frac{1}{1+y^{2}} \exp \left[-\xi / u\left(1+y^{2}\right)\right]=2 / \pi \int_{0}^{\pi / 2} \exp \left(-\xi \cos ^{2} x / u\right) \mathrm{d} x$

$$
\begin{aligned}
& =\frac{1}{\pi} \int_{0}^{\pi} \mathrm{d} \gamma \exp (-\xi / 2 u) \exp (-\xi \cos \gamma / 2 u) \\
& =\exp (-\xi / 2 u) J_{0}(i \xi / 2 u) .
\end{aligned}
$$

Using

$$
J_{0}(i \cdot x)=\sum_{\eta=0}^{\infty} \frac{x^{2 n}}{2^{2 n}(n !)^{2}}
$$

(cf. Ref. 32) and performing the integration over $u$ finally yields after some algebra the analytical expression

$$
\begin{array}{r}
P_{1}(\xi)=1-\frac{p}{2}\left[\mathrm{e}^{-\xi / 2}(1+\xi / 8)+\xi / 2 E i(-\xi / 2)+\sum_{n=2}^{\infty} \frac{(2 n-2) !}{2^{2 n}(n !)^{2}} \xi / 2 \exp (-\xi / 2) \sum_{m=0}^{2 n-2} \frac{(\xi / 2)^{m}}{m !}\right] \\
\text { for } 0 \leq \xi \leq \xi_{c} .
\end{array}
$$

For $\xi=0$ this leads again immediately to $P_{(0)}=1-\frac{1}{2} p$ as can be expected in first order approximation. We also easily see that $P_{1}(\xi)$ tends to unity when $\xi$ goes to infinity which practically occurs if $\xi$ tends to $\xi_{c}$ in the high-density case $\left(\xi_{c} \gg 1\right)$. For $\xi \gtrsim 10$ (supposing $\left.\xi_{c}>10\right)$ the asymptotic expansion of the Bessel function $J_{0}(i . x)=\mathrm{e}^{x} /(2 \pi x)^{\frac{1}{2}}(1+1 / 8 x+\ldots)$ may be used. The leading term of the asymptotic series expansion approximates the function with an error tending towards zero when $\xi$ increases. For $\xi=10$ this error is of the order of 2 per cent. So we have for $\xi \gtrsim 10$

$$
P_{1}(\xi)=1-p / 2 \int_{0}^{1} \mathrm{~d} u \exp (-\xi / 2 u) \frac{\exp (\xi / 2 u)}{(2 \pi \xi / 2 u)^{\frac{1}{2}}}=1-\frac{p}{3(\pi \xi)^{\frac{1}{2}}} .
$$

By using the power series expansion (for $\xi<10$ ) and the asymptotic expansion (for $\xi \gtrsim 10)$ we have plotted $P_{1}(\xi)$ for a Lorentz broadened resonance line in Fig. 2 . The results are believed to be accurate to 3 per cent.

Case of Doppler line profile

Again we start from equation (9) by now inserting

$$
\begin{aligned}
& \psi_{v} \mathrm{~d} v=\frac{\Delta v_{\mathrm{D}}}{2(\ln 2)^{\frac{3}{2}}} \exp \left(-y^{2}\right) \mathrm{d} y . \\
& P_{1}(\xi)=1-p / 2+p / 2 \frac{\int_{0}^{\infty} \mathrm{d} y \int_{0}^{1} \mathrm{~d} u \exp \left(-y^{2}\right)\left(1-\exp \left[-\xi \exp \left(-y^{2}\right) / u\right]\right)}{\int_{0}^{\infty} \mathrm{d} y \mathrm{e}^{-y^{2}}}
\end{aligned}
$$




$$
=1-p / 2 \frac{2}{\pi^{\frac{1}{2}}} \int_{0}^{1} \mathrm{~d} u \int_{0}^{1} \mathrm{~d} y\left(\exp \left[-\xi \exp \left(-y^{2}\right) / u\right]\right)\left(\exp \left(-y^{2}\right)\right)
$$

Expanding the expression $\exp \left[-\xi \exp \left(-y^{2}\right) / u\right]$ in a series and integrating term by term over $y$ we obtain

$$
P_{1}(\xi)=1-p / 2 \int_{0}^{1} \mathrm{~d} u \sum_{n=0}^{x}(-1)^{n} \frac{(\xi / u)^{n}}{n !(n+1)^{\frac{1}{2}}}=1-p / 2 \int_{0}^{1} \mathrm{~d} u[1-R(\xi / u)] .
$$

where the function $R(x)$ has been tabulated by MiTCHELl and ZEMANSKY ${ }^{(9)}$ and DE: GROOT $^{(3.3)}$. It should be noted that by only omitting $(n+1)^{\frac{1}{2}}$ occurring in the dominator of the series expansion we would have obtained the same expression for $P_{1}(\xi)$ as in the case with a rectangular profile.

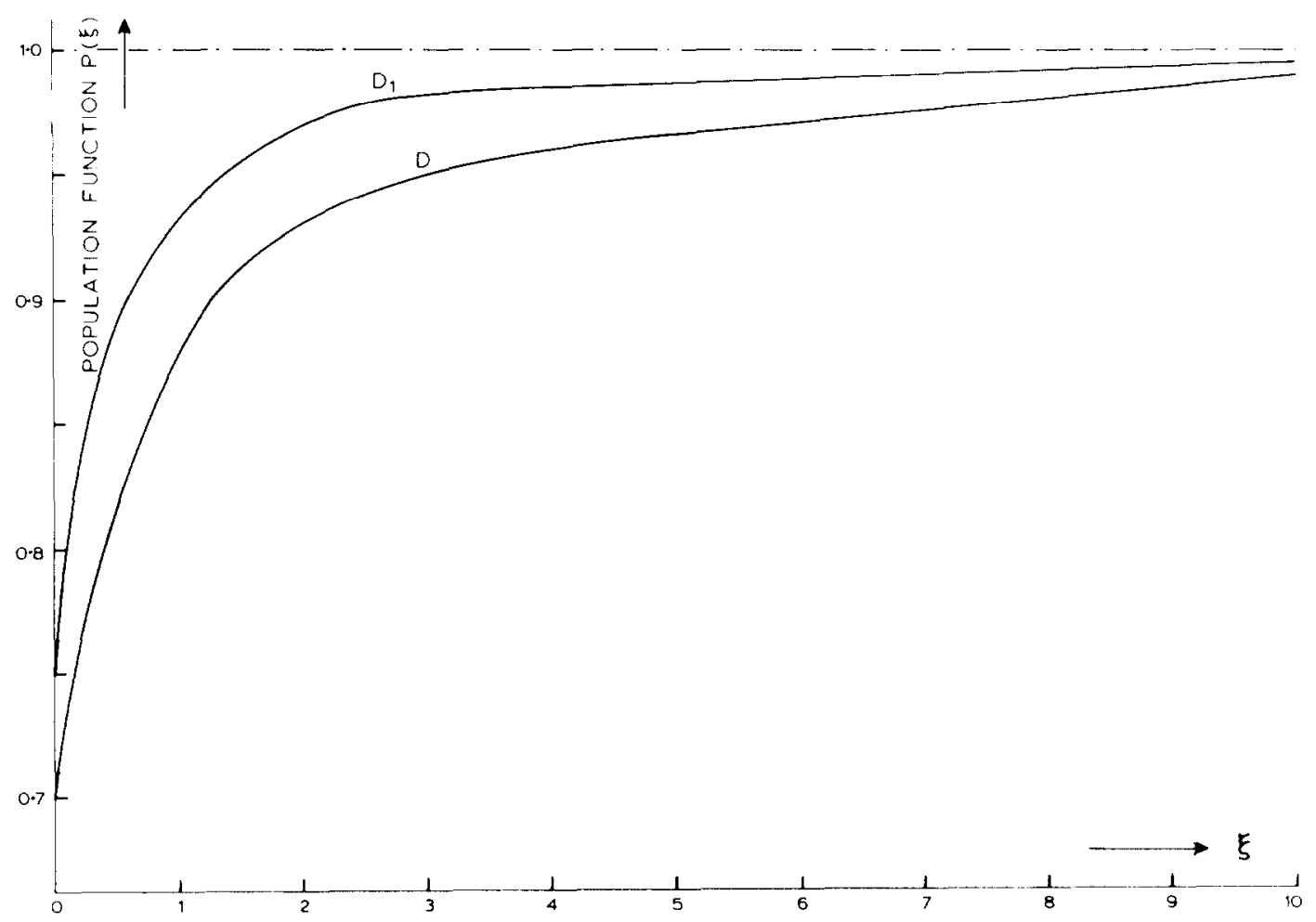

Fig. 3. First order approximation (curve $D_{1}$ ) and exact population function (curve $D$ ) for a Doppler line profile in the high density case for $p=0.5$. The exact solution was calculated by HEARN $^{(23)}$ with an electronic computer.

$P_{1}(\xi)$ is calculated by graphical integration for $p=0 \cdot 32$ and shown in Fig. 2 as a function of $\xi$. It is interesting to note in Fig. 2 that the population function for a rectangular line shape approaches that for a Doppler line shape much better than that for a Lorentz 
line shape, in particular at the more interior parts of the flame. In general the population of the excited state in case of a rectangular shape is higher than in the other cases, at least in the limit of high atomic densities. The actual population in a flame at one atmosphere will be situated in between the extreme cases of pure Lorentz and pure Doppler broadening, dependent on the value of the $a^{\prime}$-parameter of the curve-of-growth ${ }^{(9,21)}$. Figure 3 shows in the high density case the deviation of our first order approximation from the exact population function as calculated by HEARN ${ }^{(23)}$ in the case of pure Doppler broadening for the smallest value $p=0.5$ used by HEARN. As the deviation of $P_{1}(\xi)$ from the exact population function tends to zero when $p$ decreases, we may expect that the error introduced by our approximations will be less than the deviation shown in Fig. 4 , for all relevant $p$ values of our sodium coloured flames ( $\$ \leqslant 0 \cdot 3$ ).

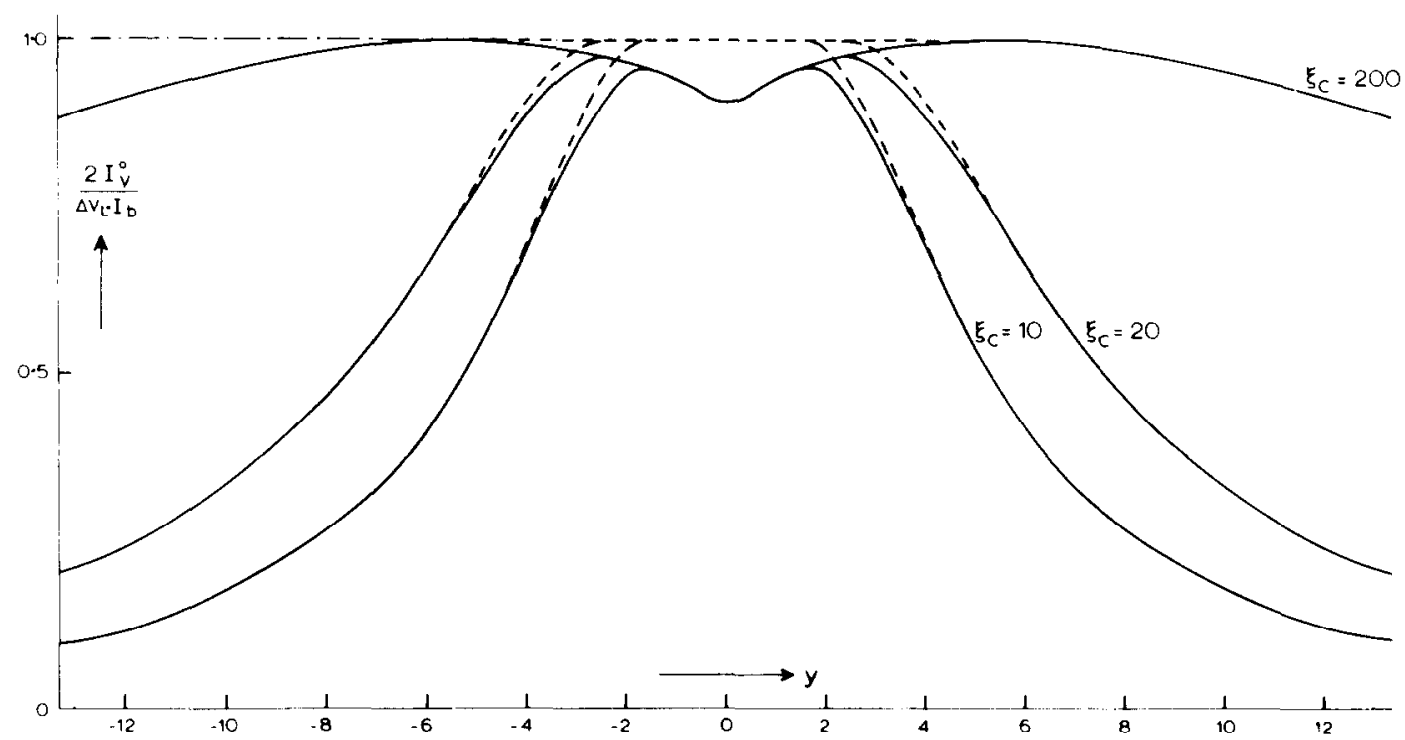

FIG. 4. Normalized intensity profiles of spectral line viewed in a direction perpendicular to the flame surface, plotted versus the dimensionless frequency difference $y$ in the case of pure Lorentz broadening. The solid curves are calculated from the first order approximation of the population function for $p=0.32$ for three values of $\xi_{c}$ corresponding to three different high atomic densities. The dashed lines represent the normalized line profiles that would be obtained if the occupation of the excited state would obey Boltzmann's distribution law at flame temperature.

The profile of the emitted self-absorbed resonance line

The line profiles of the self-absorbed resonance radiation emitted by the flame in normal direction to the flame boundary in an infinitesimal solid angle, were calculated from the population function in the high-density case. In calculating $I_{v}(0=\pi ; \xi=0)$ (denoted by $J_{v}^{0}$ ) we subdivide the flame into plane parallel slabs of breadth $\mathrm{d} \xi$, which are labelled by their dimensionless distance $\xi$ to the flame surface.

The intensity of the radiation emitted at frequency $v$ by such a subelement per unit solid angle per unit band-width and unit area, is given by $I_{h} \psi_{v} P(\xi) \mathrm{d} \xi$. Because of selfabsorption only a fraction $\exp -\left(\xi \psi_{\nu}\right)$ of this radiation is transmitted to the flame surface. 
So we obtain for the total radiation intensity emitted at frequency $v$ at the flame surface in a solid angle $\mathrm{d} \omega$ and per unit area and at normal direction

$$
I_{v}^{0} \mathrm{~d} \omega=\int_{0}^{2 \xi_{c}} I_{b} \psi_{v} P(\xi) \exp \left(-\xi \psi_{v}\right) \mathrm{d} \xi \mathrm{d} \omega .
$$

In the high-density case we may approximate $P(\xi)=1$ for $\xi_{c} \leq \xi \leq 2 \xi_{c}$ because of the small contribution to $I_{v}^{0}$ of the subelements with $\xi>\xi_{c}$.

\section{Case of rectangular profile}

For the rectangular profile we have to insert $\psi_{v}=1$ within the bandwidth of the line and $P(\xi)=P_{2}(\xi)$, where $P_{2}(\xi)$ represents the second order approximation of $P(\xi)$. This yiclds for $I_{v}^{0}$ (cf. cquation (18)) in the high-density case

$$
\begin{gathered}
I_{v}^{0} \mathrm{~d} \omega-I_{b} \mathrm{~d} \omega\left[\int_{0}^{2 \xi} \exp (-\xi) \mathrm{d} \xi-p / 2 \int_{0}^{\xi} \mathrm{d} \xi \int_{0}^{1} \mathrm{~d} s \exp [-(\xi+\xi / s)]-\right. \\
\left.\frac{p^{2}}{4} \int_{0}^{\xi} \mathrm{d} \xi \int_{0}^{1} \mathrm{~d} u \int_{0}^{1} \mathrm{~d} s\left(\frac{u}{u-s}[\exp (-\xi / u)-\exp (-\xi / s)]-\frac{u}{u+s} \exp (-\xi / u)\right) \exp (-\xi)\right] .
\end{gathered}
$$

Changing the order of integration $\xi, s$ and $u$ we finally obtain by using the inequality $\exp \left(-2 \dot{\xi}_{c}\right) \ll 1$

$$
I_{v}^{0} \mathrm{~d} \omega=I_{b} \mathrm{~d} \omega\left(1-0 \cdot 15 p-0 \cdot 10 p^{2}\right) .
$$

At given $p$-value the intensity of the line will never approach the black-body intensity, however large the metal concentration may be. As a consequence the excitation temperature, measured by line-reversal, will never approach the true translation temperature of the flame within a certain finite interval that is the closer to zero the smaller $p$ is.

Case of Lorentz line profile

When the frequency distribution $\psi_{v}$ is determined by pure Lorentz broadening, equation (18) reduces in the high-density case, when using the first order approximation of $P(\xi)$ to

$$
\begin{aligned}
I_{v}^{0} \mathrm{~d} \omega= & I_{b} \frac{\Delta v_{L}}{2}\left[\int_{0}^{2} \frac{\mathrm{d} \xi}{1+y^{2}} \exp \left[-\xi /\left(1+y^{2}\right)\right]\right. \\
& \left.-p / 2 \int_{0}^{\xi} \frac{\mathrm{d} \xi}{1+y^{2}} \exp \left[-\xi /\left(1+y^{2}\right)\right] \cdot \int_{0}^{1} \mathrm{~d} u \exp (-\xi / 2 u) . J_{0}(i \xi / 2 u)\right] \mathrm{d} \omega .
\end{aligned}
$$

Performing the integration over $\xi$ in the first term of this expression and using

$$
\int_{0}^{1} \mathrm{~d} u \exp (-\xi / 2 u) J_{0}(i \xi / 2 u)=\frac{2}{p}\left[1-P_{1}(\xi)\right]
$$


where $P_{1}(\xi)$ stands for the first order approximation of $P(\xi)$, we obtain

$$
I_{v}^{o}=I_{b} \frac{\Delta v_{\mathbf{L}}}{2}\left[1-\exp \left[-2 \xi_{c} /\left(1+y^{2}\right)\right]-\int_{0}^{\xi_{c}} \frac{\mathrm{d} \xi}{1+y^{2}} \exp \left[-\xi /\left(1+y^{2}\right)\right]\left[1-P_{1}(\xi)\right]\right] .
$$

The second term in this expression represents the shortage in total line intensity, due to radiative non-equilibrium. From equation (20) $I_{v}^{0}$ was calculated by means $0_{1}$ graphical integration for various values of dimensionless frequency $y$ with an accuracy of about 4 per cent. The results are plotted in Fig. 4 for $\xi_{c}=10,20$ and 200.

The plots show, as was also found by HEARN ${ }^{(23)}$ and others ${ }^{(24,25)}$ in the case of a Doppler broadened line shape, that the spectral distribution of the intensity exhibits a dip at the line center. Such dips are well known when a flame is surrounded by a cooler border layer which causes self-reversal of the resonance lines. In our model, however, the flame temperature is assumed to be strictly constant throughout the flame cross-section. Obviously, the dip found here is caused by the underpopulation of the excited metal atoms near to the flame border, owing to the radiative nonequilibrium. If the metal concentration in the flame (i.e. $\xi_{c}$ ) is large enough, the intensity in the wings of the broadened emission line will approach the black-body intensity. This is easily understood, if we realise that, even in the high-density case photons emitted by the flame at frequencies belonging to the line wings originate largely from the more interior parts $(\xi>2)$ where the population function approaches its equilibrium value. Photons emitted at line center, however, where the absorption coefficient has its maximum value, originate mainly from places that are within a distance $\xi=2$ from the flame border. It is just at these locations that deviations of the normalized population function from unity are found (see Fig. 2). When radiative (de)-excitation dominates collisional (de)-excitation (i.e. for large values of the yield factor $p$ ), the dip will be more pronounced.

It should be noted that we essentially used in our calculations the assumption that the frequency at which a photon is absorbed and the frequency at which it may be reemitted are completely uncorrelated, or in other words that the population function is independent of frequency. In the case of Doppler broadened resonance lines, HEARN ${ }^{(29)}$ considers low-density plasmas where most of the excited atoms undergo no collisions before spontaneously emitting a photon. Using a computer, he calculated, in the case of a Maxwellian velocity distribution of the atoms, the source function for a uniform plane, parallel plasma by applying a redistribution function, which holds when the excited atoms do not undergo a collision before radiating a photon. This calculation was restricted to the case of pure Doppler broadening. It was found by comparison that the assumption of a source function which is independent of frequency, i.e. complete redistribution of the frequencies of the emitted photons, gives line profiles and total line intensities emitted normally to the plasma, which are identical within 10 per cent. Since, however, the variation of the source function with frequency was quite large, especially near the edge of the plasma, the effect on the emitted line profile appeared to be increasingly significant for increasing angle $\theta$ with respect to the normal direction of the plane boundary.

In our flame experiments the Doppler width is of the same order of magnitude as the Lorentz width; this fact implies that the above analysis does not directly apply to resonance lines emitted by our flames. At first glance one would expect that the frequencies at which a photon is absorbed and at which it is re-emitted are much less correlated in 
llames at atmospheric pressure than in low-density plasmas. because in such flames the intercollision time is much shorter than in low-density-plasmas. This implies that an atom will suffer many collisions that change its velocity during the natural life-time $\left(=A^{-1}\right)$ of its excited state. But we have to bear in mind that the actual lifetime of the excited state $\left(=\left(k_{\ldots 1}+A\right)^{-1}=p A^{-1}\right)$ is also shortened at high species densities because of the increasing rate of quenching collisions. Since, however, the occurrence of a frequency dependence in the source function does not seem to affect markedly the calculations (as shown by HEARN ${ }^{(29)}$ ) frequency-independent source functions may be safely used under our flame conditions in the case of Lorentz and mixed line profiles (cf. Part II).

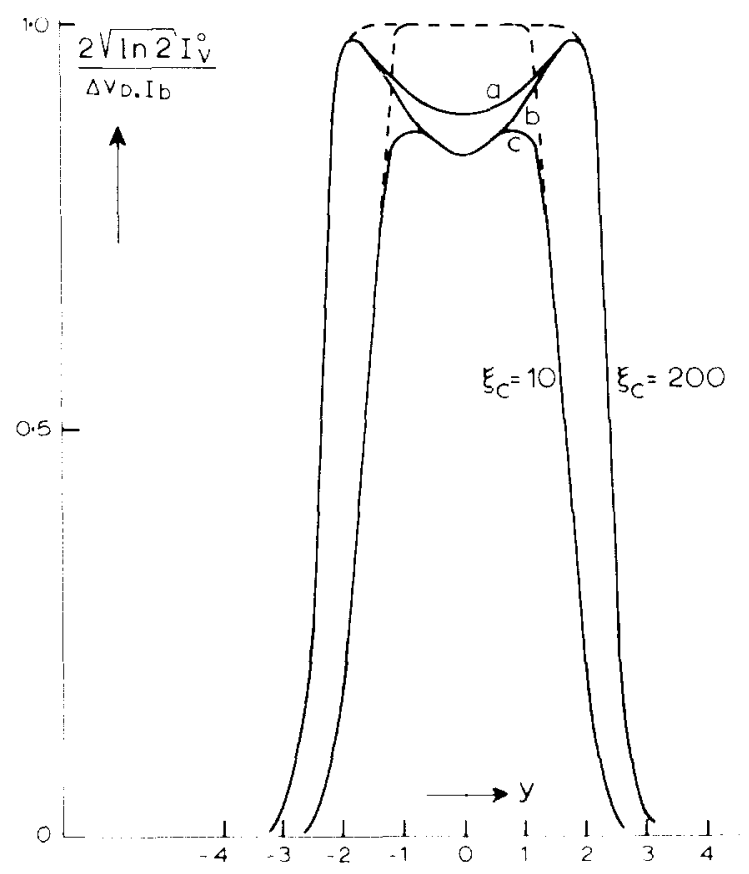

FiG. 5. Normalized intensity profiles of spectral line viewed in a direction perpendicular to the flame surface, plotted versus the dimensionless frequency difference $y$ in the case of pure Doppler broadening. Curve $a$ is calculated from our first order approximation of the population function for $\xi_{c}=200$ and $p=0.5$. The curves $b$ and $\left(\right.$ are computed by HEARN for $\xi_{c}=10$ and $\xi_{c}=200$ ) using the exact population function for $p-0.5$. The dashed lines represent the normalized lineprofiles that would be obtained if the occupation of the excited state would obey Boltzmann's distribution law at flame temperature.

\section{Case of a Doppler profile}

Applying $\psi_{v} \mathrm{~d} v=\Delta v_{\mathrm{D}} / 2(\ln 2)^{\frac{1}{2}} \exp \left(-y^{2}\right) \mathrm{d} y$ and using the first order approximation of $P(\xi)$ with respect to $p$ equation (18) changes for the high density case into 


$$
\begin{aligned}
I_{v}^{0} \mathrm{~d} \omega= & I_{b} \frac{\Delta v_{\mathrm{D}}}{2(\ln 2)^{\frac{1}{2}}}\left[\int_{0}^{2 \xi} \mathrm{d} \xi \exp \left(-y^{2}\right) \cdot \exp \left[-\xi \exp \left(-y^{2}\right)\right]\right. \\
& \left.-p / 2 \int_{0}^{\xi} \mathrm{d} \xi \exp \left(-y^{2}\right) \exp \left[-\xi \exp \left(-y^{2}\right)\right] \cdot \int_{0}^{1} \mathrm{~d} u[1-R(\xi / u)]\right]
\end{aligned}
$$

(cf. equation (17) for definition of function $R(x)$ ). $I_{v}^{0}$ was calculated as a fraction of $I_{b}$ by means of graphical integration. The results are plotted in Fig. 5 for the case $\xi_{c}=200$ and $p=0.5($ curve $a)$.

\section{Integrated (total) line intensity}

In emission flame spectroscopy one usually measures the line intensity integrated over its frequency band-width ("total line intensity"). It is this quantity that determines the value of the line reversal temperature measured and that is plotted in emission "curves-of-growth". In particular, we are interested here in the calculation of the shortage in total line intensity, when compared to conditions where the occupation of the excited state obeys the Boltzmann distribution law. The fraction of this shortage (corresponding to the area between the solid and dashed curves of Figs. 4 and 5) when compared to the Boltzmann equilibrium intensity (corresponding to the area under the dashed curves in Figs. 4 and 5) will be denoted by $\alpha$. Here $\alpha$ is a function of the atomic density in the flame and thus of $\xi_{c}=\kappa_{m} \mathrm{~L}$ (the flame dimensions being held constant). Hence we shall derive here the dependence of $\alpha$ on $\xi_{c}$ in the high-density case, for the three typical line profiles considered previously.

The total line intensity per unit solid angle in a direction perpendicular to the flame surface follows by integrating $I_{v}^{0}$ with respect to frequency. In the high-density case we computed $\int_{0}^{\infty} I_{v}^{0} \mathrm{~d} v$ for the three different line shapes, using the approximate population functions.

\section{Case of rectangular line profile}

We have from equation (19)

$$
\int_{0}^{\infty} I_{v}^{0} \mathrm{~d} v=I_{b} \Delta v\left(1-0 \cdot 15 p-0 \cdot 10 p^{2}\right)=I_{b} \Delta v(1-\alpha)
$$

Here, of course,

does not depend on $\xi_{c}$.

$$
\alpha=0 \cdot 15 p+0 \cdot 10 p^{2}
$$

\section{Case of Doppler line profile}

We have from equation (21)

$$
\int_{0}^{\infty} I_{v}^{0} \mathrm{~d} v=I_{b} \frac{\Delta v_{\mathrm{D}}}{(\ln 2)^{\frac{1}{2}}}\left(2 \xi_{c} \frac{\pi^{\frac{1}{2}}}{2} S\left(2 \xi_{c}\right)-\frac{p}{2} \int_{0}^{c_{c}} \mathrm{~d} \xi^{\frac{\pi^{\frac{1}{2}}}{2}}[1-R(\xi)] \int_{0}^{1} \mathrm{~d} u[1-R(\xi / u)]\right),
$$


where

$$
\xi S(\xi) \equiv \frac{2}{\pi^{\frac{1}{2}}} \int_{0}^{\infty}\left(1-\exp \left[-\xi \exp \left(-y^{2}\right)\right] \mathrm{d} y\right.
$$

is tabulated by Mitchell and Zemansky. ${ }^{(9)}$ On account of the definition of $\alpha$ we also have

$$
\int_{0}^{\infty} I_{v}^{\mathrm{n}} \mathrm{d} v=I_{b} \frac{\Delta v_{\mathrm{D}}}{(\ln 2)^{\frac{1}{2}}} \frac{\pi^{\frac{1}{2}}}{2} 2 \xi_{c} S\left(2 \xi_{c}\right)\left[1-\alpha\left(\xi_{c}\right)\right]
$$

where

$$
\frac{\Delta v_{\mathrm{D}}}{(\ln 2)^{\frac{1}{2}}} \frac{\pi^{\frac{1}{2}}}{2} 2 \xi_{c} S\left(2 \xi_{c}\right)=A_{G} / 2 \pi
$$

represents the integrated absorption factor in the thermodynamic equilibrium ("Total Absorption") and the factor $1-\alpha\left(\xi_{c}\right)$ describes the effect of non-thermal population of the excited level. Comparison of both expressions yields

$$
\alpha\left(\xi_{c}\right)=\frac{\frac{1}{2} p \int_{0}^{\xi_{c}}[1-R(\xi)] \int_{0}^{1}[1-R(\xi / u)] \mathrm{d} u \mathrm{~d} \xi}{2 \xi_{c} S\left(2 \xi_{c}\right)}=\frac{\int_{0}^{\xi_{c}}[1-R(\xi)]\left[1-P_{1}(\xi)\right] \mathrm{d} \xi}{2 \xi_{c} S\left(2 \xi_{c}\right)}
$$

where $P_{1}(\xi)$ represents the first order approximation of the population function in the case of a pure Doppler line profile.

When the occupation of excited state is in thermal equilibrium, we have $P(\xi)=1$ and then indeed $\alpha\left(\xi_{c}\right)=0$ as may be expected. For coordinate values $\xi \gtrsim 10$,

$$
\int_{0}^{1}[1-R(\xi / u)] \mathrm{d} u<[1-R(\xi)]<\frac{1}{2} \xi^{-1}
$$

is valid and consequently,

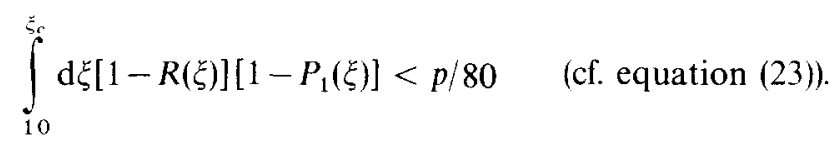

This implies that for $p=0.5$,

$$
\int_{10}^{\xi_{c}}[1-R(\xi)]\left[1-P_{1}(\xi)\right] d \xi
$$

amounts to at most 2 per cent of the remaining part of the integral:

$$
\int_{0}^{10}[1-R(\xi)]\left[1-P_{1}(\xi)\right] \mathrm{d} \xi
$$


occurring in the numerator of the expression for $\alpha\left(\xi_{c}\right)$. Moreover the function $S(\xi)$ increases very slowly with $\xi$ in the range of high $\xi$-values (its asymptotic behaviour is similar to that of $(\ln \xi)^{\frac{1}{2}}$ (cf. Ref. 34) so that we may conclude that for a pure Doppler profile $\alpha\left(\xi_{c}\right)$ is a very slowly decreasing function of the metal concentration (e.g. when $\xi_{c}$ rises from 10 to 200 for a $p$ value of $0.5, \alpha\left(\xi_{c}\right)$ is found to decrease from 0.10 to 0.07 (see Fig. 5).

Figure 5 also shows the results of HEARN's exact calculations ${ }^{(23)}$ on lineprofiles in the case of pure Doppler broadening for $p=0.5$ at two large values of the metal concentration $\xi_{c}=10$ and $\xi_{c}=200$ (curve $b$ and $c$ ). From equation (22) it follows that the use of the first order approximation $P_{1}(\xi)$ for a rectangular lineprofile leads to a shortage in total line-intensity for high metal densities at $p=0.5$, that deviates by 25 per cent from the shortage calculated with the aid of the second order approximation $P_{2}(\xi)$. The close resemblence between the rectangular and pure Doppler case (see Fig. 2) leads us to expect also for a pure Doppler lineprofile a similar systematic deviation between the intensity shortage calculated in first order approximation and that calculated in second order approximation. Indeed, correcting our lineshape calculations in first order approximation for pure Doppler broadening by the amount of 25 per cent, the shortage in total line intensity is then found to deviate by only 2 per cent from the exact value computed by Hearn (see Fig. 5). Assuming that the procedure of estimating the systematic error introduced in our first order approximation is also valid for $p$-values less than 0.5 , we may expect from equation (22) that our calculations on $\alpha$-values by using first order approximations of the population function are valid with a maximum error of 15 per cent, if $p \leq 0 \cdot 3$.

\section{Case of Lorentz broadening}

Using in this case

$$
\int_{0}^{\infty} \frac{1}{1+y^{2}} \exp \left[-\xi /\left(1+y^{2}\right)\right] \mathrm{d} y=J_{0}(i \xi / 2) \exp (-\xi / 2)
$$

we get

$$
\int_{0}^{\infty} I_{v}^{0} \mathrm{~d} v=I_{b} \Delta v_{\mathrm{L}}\left[\int_{0}^{2 \xi_{c}} \mathrm{~d} \xi J_{0}(i \xi / 2) \exp (-\xi / 2)-p / 2 \int_{0}^{\xi_{c}} J_{0}(i \xi / 2) \exp (-\xi / 2) B(\xi) \mathrm{d} \xi\right],
$$

where

$$
B(\xi)=\int_{0}^{1} \mathrm{~d} u \exp (-\xi / 2 u) J_{0}(i . \xi / 2 u) \mathrm{d} u=2 / p\left(1-P_{1}(\xi)\right)
$$

Applying

$$
\int_{0}^{2 \xi_{c}} \mathrm{~d} \xi \exp (-\xi / 2) J_{0}(i \cdot \xi / 2) \approx 2\left(\pi \xi_{c}\right)^{\frac{1}{2}}
$$

which holds for $\xi_{c} \gtrsim 5$ with an accuracy to 2 per cent, we find for the total emission 
intensity in the case of full Boltzmann equilibrium: $I_{h} \Delta v_{\mathrm{L}} 2\left(\pi \xi_{c}\right)^{\frac{1}{2}}$ for high densities. This function increases as the square root of the density, which is a well known feature of the curve-of-growth for Lorentz line shape. Comparing this expression with the above expression for $\int_{0}^{\infty} I_{v}^{0} \mathrm{~d} v$ we find for $\alpha\left(\xi_{c}\right)$ in the high-density case

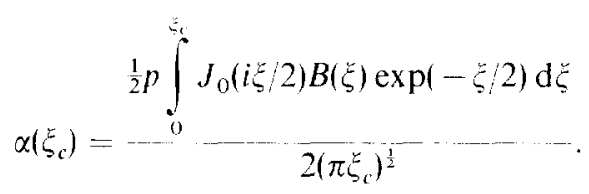

Integrating the numerator by parts for $\xi_{c} \geq 10$ one gets

$$
\frac{1}{2} p \int_{0}^{10} J_{0}\left(i \xi_{2}\right) \exp (-\xi / 2) B(\xi) \mathrm{d} \xi+\int_{i_{0}}^{t_{0}} 3 \pi \xi \mathrm{d} \xi_{c} .
$$

since for $\xi_{c}>10$.

$$
J_{0}(i \xi / 2) \exp (-\xi / 2)=\frac{1}{(\pi \xi)^{\frac{1}{2}}}, \quad \text { and } \quad B(\xi)=\frac{2}{3(\pi \xi)^{\frac{1}{2}}}
$$

is valid. After integrating the latter term with respect to $\xi$ we find

$$
\alpha\left(\xi_{c}\right)=\frac{p / 2 \int_{0}^{10} J_{0}(i \xi / 2) \exp (-\xi / 2) B(\xi) \mathrm{d} \xi+\frac{2}{3 \pi} \ln \left(0 \cdot 1 \xi_{c}\right)}{2\left(\pi \xi_{c}\right)^{\frac{1}{2}}} .
$$

Since the numerator in this expression varies only very slowly with $\xi_{c}$ in the high-density case, we may conclude that here $\alpha\left(\xi_{c}\right)$ behaves asymptotically as $1 /\left(\xi_{c}\right)^{\frac{1}{2}}$, where $\xi_{c}$ is, again, proportional to the density of ground state atoms. In this respect the case of a Lorentz line shape typically differs from the two other cases considered. Also for a combined Lorentz and Doppler line profile, which is to be expected with the alkali lines in our flames, the same asymptotic behaviour according to $1 /\left(\xi_{c}\right)^{\frac{1}{2}}$ is likely to occur. It is well known that in this combined case also the thermal equilibrium emission which is proportional to the denominator in the fractional expression for $\alpha$. varies asymptotically as $\left(\xi_{\mathrm{c}}\right)^{\frac{1}{2}}$ [cf. Ref. 35].

\section{Deviation of line reversal temperature from true flame temperature}

Because of the above described effects of radiative non-equilibrium on the occupation of the excited state the line reversal temperature $T_{e}(=$ excitation temperature) will differ by an amount $\Delta T$ from the true flame temperature $T_{t}(=$ translational temperature). In the limiting case of low metal densities this discrepancy, here represented by $\Delta T_{\mathrm{L}}$ follows from

$$
N_{1}=g_{1} / g_{0} N_{0} \exp \left[-h v_{0} / k\left(T_{t}-\Delta T_{\mathrm{L}}\right)\right]
$$

Using equation (1), which relates $N_{1} / N_{1}^{0}$ to $p$ in the case that excitation by photonabsorption can be disregarded, we obtain 


$$
\frac{1}{1-p}=\exp \left[h v_{0} \Delta T_{\mathrm{L}} / k\left(T_{t}-\Delta T_{\mathrm{L}}\right) T_{t}\right]
$$

which results into

$$
\Delta T_{\mathrm{L}}=\frac{k T_{t}^{2}}{h v_{0}} \ln (1-p)^{-1} /\left[1+\frac{k T_{t}}{h v_{0}} \ln (1 \quad p)^{-1}\right] \approx \begin{gathered}
k T_{t}^{2} \\
h v_{0}
\end{gathered} \ln (1-p)^{-1}
$$

since for all relevant $p$-values in our flames $\left(k T_{t} / h v_{0}\right) \ln (1-p)^{-1} \ll 1$. For small $p$-values we have, in good approximation, $\Delta T_{\mathrm{L}}=p\left(k T_{t}^{2} / h v_{0}\right)$. Of course, this expression does not depend on the frequency distribution of the absorption coefficient, because virtually all generated light quanta will escape the flame before being reabsorbed.

In general the integrated intensity of the emission line can be rewritten as

$$
\int_{0}^{\infty} I_{v}^{0} \mathrm{~d} v=I_{b}\left(v_{0}\right)\left[1-\alpha\left(\xi_{c}\right)\right] . A_{G} / 2 \pi
$$

where $I_{b}$ represents the radiation intensity per unit band-width for a black body at temperature $T_{t}$. and $A_{\mathrm{c}} / 2 \pi$ is the "total absorption" of the flame under equilibrium condition (cf. Mitchell and ZEMANSKY ${ }^{(19)}$ ). This "total absorption" is obtained by integrating the absorption factor with respect to frequency. The factor $\left[1-\alpha\left(\xi_{c}\right)\right]$ accounts for the effect of the uncerpopulation of the excited state. Disregarding the (weak) temperature dependence of the integrated absorption factor, we find the deviation $\Delta T$ in the measured line-reversal temperature from

$$
\exp \left[-h v_{0} / k T_{t}\right]\left[1-\alpha\left(\xi_{c}\right)\right]=\exp \left[-h v_{0} / k\left(T_{t}-\Delta T\right)\right]
$$

which yields

$$
\Delta T-\frac{\left(k T_{t}^{2} / h v_{0}\right) \ln \left[1-\alpha\left(\xi_{c}\right)\right]^{-1}}{1+\left(k T_{l} / h v_{0}\right) \ln \left[1-\alpha\left(\xi_{c}\right)\right]^{-1}} .
$$

By neglecting $k T_{t} / h v_{0} \ln \left[1-\alpha\left(\xi_{c}\right)\right]^{-1}$ with respect to unity and by series expansion of $\ln \left[1-\alpha\left(\xi_{c}\right)\right]^{-1}$ one finds

$$
\Delta T=\frac{k T_{t}^{2}}{h v_{0}} \alpha\left(\xi_{c}\right)
$$

This approximate expression holds in the high-density case $\left(\xi_{c} \gtrsim 5\right)$ for all measured values of the yield factor $p$ (see Part II) and for all line profiles treated here.

In the case of a rectangular line profile, $\alpha\left(\xi_{c}\right)$ tends to the finite value $\left(0 \cdot 15 p+0 \cdot 10 p^{2}\right)$ in the limit of high-densities (cf. equation (22)). When the metal density varies from small to large values the absolute deviation in line-reversal (or excitation) temperature decreases monotonously from the asymptotic value $\left(k T_{1}^{2} / h v_{0}\right) \ln (1-p)^{-1}$ (cf. equation (26)) to the asymptotic value $\left(k T_{t}^{2} / h v_{0}\right)\left(0 \cdot 15 p+0 \cdot 10 p^{2}\right)$. The true flame temperature is never approached in this case.

In the more realistic case of combined Lorentz and Doppler broadening that is expected to occur with the alkali lines in our flames, $\alpha\left(\xi_{c}\right)$ approaches zero as $1 /\left(\xi_{c}\right)^{\frac{1}{2}}$ in the high-density case (cf. above). Accordingly, the deviation $\Delta T$ in line-reversal temperature approaches zero as $1 /\left(N_{0}\right)^{\frac{1}{2}}$, where $N_{0}$ is the concentration of ground state atoms in 
the flame. This asymptotic bchaviour enables us to find the true flame temperature by extrapolation from the reversal temperatures measured at high concentrations (cf. Part II). By measuring reversal temperatures when the atomic density varies from small values where $\Delta T=\Delta T_{\mathrm{L}}=\left(k T_{t}^{2} / h v_{0}\right) \ln (1-p)^{-1}$, to large values where $\Delta T$ tends to zero, we are also able to derive or to check the yield factor $p$ of resonance fluorescence (cf. Part II; cf. also Refs. 7 and 15). Moreover, the knowledge of the variation of line-reversal temperature as a function of the metal concentration in the flame enables us to correct the curve-of-growth measured in emission when its shape has been affected by the lack in radiative equilibrium (cf. Part II). The experimental emission values plotted in the curveof-growth can be simply corrected for this lack in equilibrium by multiplying them by $(1-\alpha)^{-1}$ where the fractional shortage $\alpha$ in the total line intensity is related to the deviation $\Delta T$ of the line reversal temperature through equation (28). This correction may be essential when values of the so called $a^{\prime}$-parameter and atomic metal content are to be derived from the curve-of-growth at relatively high $p$-values which may occur in flames with noble gas as diluent gas (cf. Part II). It should be noted, however, that when using the so-called duplication curve in the determination of $a^{\prime}$ (Refs. 21 and 42) one is not troubled by lack in equilibrium radiation.

\section{Limitations by diffusion effect}

In the foregoing derivations a constant density of ground state atoms was assumed throughout the flame volume coloured by metal vapour. However, outward diffusion of the atoms into the (colourless) flame mantle (cf. Part II) will cause a gradual decrease of atomic density from the maximum value $N_{0}$ (at the flame centre) to zero (at the flame border). The derived expressions will be valid in good approximation only if the thickness of the layer where the major drop in density, because of diffusion occurs, is small when compared to $\kappa_{m}^{-1}$. This limitation is more severe for a rectangular line profile where $\kappa_{v}=\kappa_{m}$ for any $v$ within the line band-width, than for a Lorentzian profile where a noticeable part of the radiation occurs at frequency $v$ for which $\kappa_{v}<\frac{1}{2} \kappa_{m}$. VAN SUCHTELEN $^{(36)}$ has derived an expression for the drop in atomic density caused by diffusion in the one-dimensional case of an isothermal infinite slab of flame gases with the central plane at $x=0$, assuming that the flame part for which $x>0$ is homogeneously fed at the base (i.e. $z=0$ ) by a constant supply of metal salt. According to his derivation the atomic density $N_{0}(x)$ is described by

$$
N_{0}(x)=\frac{1}{2} N_{0}\left[1+\operatorname{erf}\left(x(4 D Z / v)^{-\frac{1}{2}}\right)\right] \text { for } x>0
$$

and

$$
N_{0}(x)=N_{0}-N_{0}(-x) \text { for } x<0
$$

where $N_{0}$ is the maximum density at $x \rightarrow+\infty$ and $z$ is the vertical distance over which the flame gases have travelled with a constant velocity $v$ after leaving the burner. $D$ represents the diffusion constant of the atomic metal vapour which is present as a trace in the flame gases. In these expressions the error function may be approximated by

$$
\operatorname{erf} x=\frac{2}{\pi^{\frac{1}{2}}} \int_{0}^{x} \mathrm{~d} u \exp \left(-u^{2}\right) \approx x
$$


for $0 \leq x \leq 1$, and erf $x=1$ for $x>1$. Using this approximation we may define the characteristic thickness of the layer where diffusion affects noticeably the atomic density by $4(D z / v)^{\frac{1}{2}}$. We shall consider this limitation in more detail in Part II.

\section{Line broadening effect of quenching collisions}

The increased de-excitation probability per sec of excited metal atoms due to quenching collisions entails a decrease in radiative lifetime of the emitting atoms and consequently a broadening of the line width $\Delta v_{t}$. Denoting, as before, the optical transition probability by $A$ and the rate of de-excitation collisions by $k_{-1}$, we have simply

$$
\Delta v_{t}=\left(A+k_{-1}\right) / 2 \pi=A / 2 \pi p
$$

where $p$ is again the yield factor of resonance fluorescence. For small $p$-values as they occur mostly in flames at $1 \mathrm{~atm}$ pressurc, $\Delta v_{t}$ may be of comparable magnitude as the Lorentz broadening $\Delta v_{\mathrm{L}}$ caused by adiabatic collisions with flame molecules. Assuming that the shape of a spectral line broadened by quenching collisions is the same as that of Lorentz broadened line we have for the $a^{\prime}$-parameter describing the curve-of-growth ${ }^{(9)}$

$$
a^{\prime}=\frac{A / 2 \pi p+\Delta v_{\mathrm{L}}}{\Delta v_{\mathrm{D}}} \cdot(\ln 2)^{\frac{1}{2}},
$$

where $\Lambda v_{L}$ represents the Lorentz hroadening and $\Delta v_{D}$ the effect of Doppler broadening. When we compare the measured $a^{\prime}$-values with theoretical values calculated on the basis of Weiskopf-Lindholm theory, the effect of quenching broadening should be taken into account. ${ }^{(20,37,38)}$

Velocity-dependent quenching cross-section calculated from temperature-dependent quenching rate

In our flame measurements of quenching rates we are concerned with quenching collisions between excited atoms and flame molecules which have a maxwellian velocity distribution at flame temperature $T$. Since the quenching cross-section $\sigma$ may in general, be a function of the relative velocity of approach we have for the quenching rate at temperature $T$ (cf. Ref. 39) (supposing that only one species of flame molecules is effective)

$$
k_{-1}(T)=n \int_{0}^{\infty} v_{r} \sigma\left(v_{r}\right) F\left(v_{r}\right) \mathrm{d} v_{r}=n \int_{0}^{\infty} v_{r} \sigma\left(v_{r}\right)\left(\frac{\mu}{k T}\right)^{3 / 2}\left(\frac{2}{\pi}\right)^{1 / 2} v_{r}^{2} \exp \left(-\frac{1}{2} \mu v_{r}^{2} / k T\right) \mathrm{d} v_{r}
$$

where $v_{r}=$ the relative velocity of approach of the colliding species, $F\left(v_{r}\right) \mathrm{d} v_{r}=$ the probability at temperature $T$, that the relative velocity lies between $v_{r}$ and $v_{r}+\mathrm{d} v_{r}, \sigma\left(v_{r}\right)$ $=$ the cross-section for quenching collisions as function of the relative velocity, $\mu=$ the reduced mass of the colliding particles, $n=$ the density of the quenching flame molecules considered.

If we suppose that $\sigma\left(v_{r}\right)=$ constant $(=\sigma)$ we obtain $k_{-1}(T)=n \bar{v}_{r} \sigma$ where the mean relative velocity $\bar{v}_{r}=(8 k T / \pi \mu)^{1 / 2}$. If, however, we suppose that $\sigma\left(v_{r}\right)$ decreases with increasing $v_{r}$ and $\sigma\left(v_{r}\right)$ in first approximation may be represented by

$$
\sigma\left(v_{r}\right)=\frac{c}{v_{r}^{q}} .
$$


In the relevant range of $r_{r}$-values, the expression is transformed into

$$
k_{-1}(l)=\left(\frac{\mu}{k T}\right)^{3 / 2}\left(\begin{array}{l}
\pi \\
2
\end{array}\right)^{1 / 2} \int_{i}^{x} \frac{c}{l_{r}^{q}} v_{r}^{3} \exp \left(-\beta^{2} v_{r}^{2}\right) \mathrm{d} v_{r}
$$

Substituting $\beta v_{r}$ by $y$ one gets

$$
\begin{aligned}
k_{-1}(T) & =n c\left(\frac{\mu}{k T}\right)^{3 / 2}\left(\begin{array}{l}
2 \\
\pi
\end{array}\right)^{1: 2} \frac{1}{\beta^{4-q}} \int_{0}^{1} y^{3-q} \exp \left(-y^{2}\right) \mathrm{d} y \\
& =n \bar{v}_{r} \overline{\bar{v}}_{r}^{q}\left(\begin{array}{l}
4 \\
\pi
\end{array}\right)^{q / 2} \Gamma(2-q / 2) \equiv \overline{\bar{v}_{r}} \frac{c}{\bar{v}_{r}^{q}} f(q)
\end{aligned}
$$

if we use

$$
\int_{0}^{1} y^{3} \cdots \exp \left(-y^{2}\right) \mathrm{d} y=\frac{1}{2} \Gamma\left(\frac{3-q+1}{2}\right)=\frac{1}{2} \Gamma^{-}(2-q 2) .
$$

The dimensionless function $f(q)$ is independent of $T$ and $\mu$ whereas $f(0)=f(1)=1$ and $f(2)=4 / \pi=1 \cdot 27$. Furthermore we have $1 \leq f(q) \leq 4 / \pi$ for $1 \leq q \leq 2$. For $0 \leq q \leq 1$ it holds that $f(q) \leq 1$ but $f(q)$ deviates from unity by less than $0 \cdot 04$. If we introduce the effective cross-section for quenching collisions $S(T)$ at temperature $T$ defined by

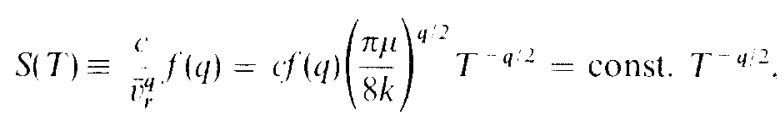

the collision frequency for quenching collisions $k_{-1}$ finally can be written as $k_{-1}-n \bar{v}_{r} S(T)$. This expression shows, that a determination of $k_{-1}$ as function of temperature may inform us about the dependence of $\sigma$ on relative velocity $v_{r}$, provided that $\sigma\left(v_{r}\right)$ can be approximated by equation (3) in the relevant range of $v_{r}$ values (cf. Part II). When the metal atoms undergo quenching collisions in a mixture of flame molecules of different species the quenching frequency is given by

$$
k_{-1}=\sum_{j=1}^{m} n_{j} \bar{v}_{r, j} \frac{c_{j}}{\bar{v}_{r, j}} f\left(q_{j}\right)=\sum_{j=1}^{m} n_{j} \bar{v}_{r, j} S_{j}(T)
$$

where $j$ indicates the various types of flame molecules. The problem of how to obtain specific information on $c_{j}$ and $q_{j}$ from measurements of $p$-values in flames of different mixture and temperature will be dealt with in Part II.

\section{Effect of radiative non-equilibrium on intensity ratio of doublet lines}

Since the resonance defect $\Delta E$ between the excited atomic level and the nearest vibrational level of the de-exciting molecule will be different for the two doublet lines of alkali atoms, a difference in radiative nonequilibrium and consequently a deviation of their equilibrium intensity ratio from $2: 1$ might result. For the yellow sodium doublet and both the red and blue potassium doublets, this discrepancy in resonance defect may amount to 0.002 .0 .007 and $0.002 \mathrm{eV}$ respectively. Since $0.002 \mathrm{eV}$ is of the order of 1 per cent when compared with $k T$ for the flames under consideration, no discrepancies between 
the doublet lines of sodium at $5890 / 96 \AA$ and potassium at $4044 / 47 \AA$ are expected to occur. For the red potassium doublet as well, we measured the same line reversal temperature with each of the doublet components in $\mathrm{H}_{2}-\mathrm{O}_{2}-\mathrm{Ar}$ flames, where considerable deviations of line reversal temperature from the true flame temperature occur (cf. Part II). This outcome is consistent with other experiments reported in the literature ${ }^{(41)}$ in which $\mathrm{K}$ vapour in an atmosphere of Ar atoms was irradiated by only one of the two components of the red $\mathrm{K}$ doublet-lines. In these experiments resonance fluorescence radiation was observed with both doublet lines, while their intensity ratio approached the theoretically predicted value at Ar pressures above $25 \mathrm{~cm} \mathrm{Hg}$. Obviously at low pressures Ar atoms are very effective in establishing an equilibrium distribution between the occupation of the $\mathrm{K} 4^{2} P_{3 / 2}$ and $4^{2} P_{1 / 2}$ levels by collisions. It is believed that, in our flames at 1 atm pressure, similar effective collisions bring about an equilibrium distribution between these levels, notwithstanding the fact that the $P$-state as a whole is underpopulated because of radiative non-equilibrium. These considerations suggest that the experiments to be described in Part II need not be made with each doublet component separately.

\section{NOTATION}

4 transition probability per sec of the considered optical transition

$A_{G} / 2 \pi \quad$ total absorption (i.e. absorption factor integrated with respect to frequency)

a parameter of the curve of growth

$\Delta E \quad$ resonance defect (i.e. energy difference between atomic excitation level and the nearest vibra-

tional level of the quenching molecule)

$I_{v}(\xi, \theta) \quad$ intensity of radiation at frequency $v$ that is incident on an infinitesimal element of volume at distance $\xi$ from the flame border under an angle of incidence $\theta$ (per unit solid angle and per frequency band width)

$\begin{array}{ll}I_{v}^{0} & I_{v}(\xi=0, \theta=\pi) \\ I_{b} & I_{v}(\xi, \theta) \text { for black-body radiation }\end{array}$

$k_{1} \quad$ probability per sec of excitation by collisions

$k_{-1} \quad$ probability per sec of quenching collisions

$2 \mathrm{~L} \quad$ thickness of flame with plane parallel boundaries

$N_{0} \quad$ number of ground state atoms per $\mathrm{cm}^{3}$

$N_{1} \quad$ number of excited atoms per $\mathrm{cm}^{3}$

$N_{1}^{0} \quad$ number of excited atoms per $\mathrm{cm}^{3}$ in case of full equilibrium

$n_{j}$ number per $\mathrm{cm}^{3}$ of quenching particles of species $j$

$p=\mathrm{A}\left(\mathrm{A}+k_{-1}\right)$ yield factor of resonance fluorescence

$P(\xi) \quad$ population function ("source function")

$S$

$T_{t}$

$T_{\mathrm{e}}$

$\Delta T=T_{t}-T_{e}$

$\Delta T_{\mathrm{L}}$

$v$

$v_{r}$

$\bar{v}$

$x$

$z$

$x$

$\dot{\delta}$

$\theta$

$\kappa_{v}$

$\kappa_{m}$

$\mu$

$v$

$v_{0}$ effective quenching cross-section averaged over Maxwellian velocity distribution of colliding particles

translational temperature (= true temperature) of the flame

excitational temperature of considered atomic excitation level (= line-reversal temperature)

deviation of line-reversal temperature from true flame temperature

$\Delta T$ in the limiting case of low metal concentrations

flow velocity of upwards streaming flame gases

relative velocity of approach of (excited) atom and quenching particle

mean value of $v_{\text {r }}$

distance of considered point in the flame to plane flame boundary at the observer's side vertical distance of considered point in flame to burner head

fractional intensity defect in outgoing radiation when compared to Boltzmann equilibrium fractional defect in excitation rate by photon absorption when compared to Boltzmann equilibrium

angle with respect to normal direction of flame boundary

absorption coefficient at frequency $v$

peak absorption coefficient (at central frequency $v_{0}$ )

reduced mass of the colliding species in the quenching process

frequency

central frequency of considered spectral line 


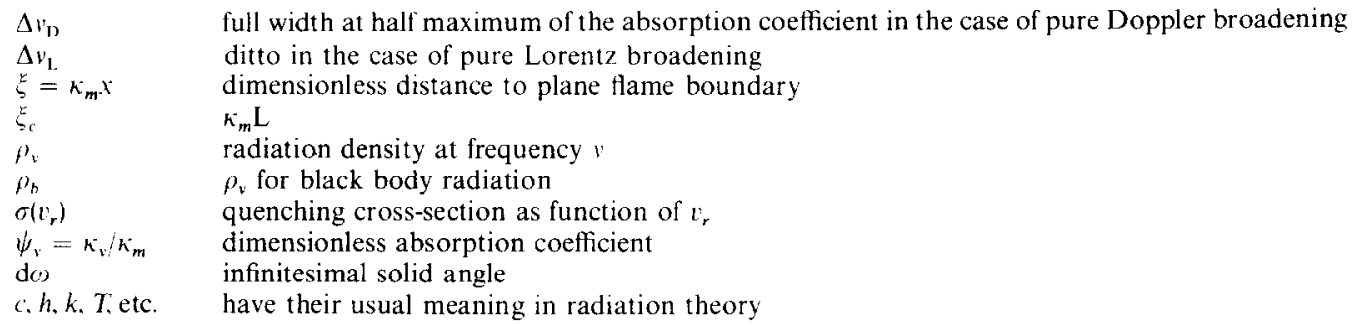

\section{REFERENCES}

1. C. Th. J. Alkemade, Colloquium Spectroscopicum Internationale, Washington, Spartan Books (1963).

2. P. T. Gilbert, Colloquium Spectroscopicum Internationale, Washington, Spartan Books (1963).

3. J. D. Winefordner and T. I. Vickers, Anal. Chem. 36, 161 (1964).

4. A. G. Gaydon and I. R. Hurle, Symposium on Combustion, 8th Pasadena Calif. 1960, 309 (1962).

5. R. C. Millikan and D. R. White, J. chem. Phys. 39, 98 (1963).

6. I. R. Hurle, J. chem. Phys. 41, 12 (1964).

7. S. Tsuchiya, J. chem. Soc. Japan 37, 6 (1964).

8. W. L. Starr. J. chem. Phys. 43, 73 (1965).

9. A. C. G. Mitchell and M. W. Zemansky, Resonance Radiation and Excited Atoms, 2nd Ed. Cambridge, (1961).

10. H. Beutler and B. Josephy, Z. phys. Chem. B8, $231 ; 403$ (1930).

11. R. Mankopff, Z. Phys. 36, 315 (1926).

12. L. V. Hamos, Z. Phys. 74, 379 (1932).

13. R. G. W. Norrish and W. Smith, Proc. R. Soc. 176A, 295 (1945).

14. W. Demtröder, Z. Phys. 166, 42 (1962).

15. E. Hulpke, E. Paul und W. Paul, Z. Phis. 177, 257 (1954).

16. A. G. Gaydon and J. R. Hurle, Symposium on Combustion, Williams and Wilkens, (1962).

17. G. Karl and G. C. Polanyi, J. chem. Phys. 38, 271 (1963).

18. P. G. Dickens, J. W. Linnett and O. Sovers, Discuss. Faraday Soc. 33, 52 (1962).

19. W. SNelleman, Thesis Utrecht, 1965.

20. P. J. Th. Zeegers and C. Th. J. Alkemade, Symposium on Combustion, Comb. Inst. Pittsburgh (1964).

21. T. Hollander, Thesis Utrecht. 1964.

22. A. L. Boers, C. Th. J. Alkemade and J. A. Smit, Physica 22, 358 (1956).

23. A. G. Hearn, Proc. phys. Soc. 81, 648 (1963).

24. S. Cuphrmann. F. Engelmann and J. Oxenius, Physics Fluids 6, 108 (1963).

25. S. Cupermann, F. Engelmann and J. Oxenius, Physics Fluids 7, 428 (1964).

26. V. A. Ambartsumyan, Theoretical Astrophysics, Pergamon Press, Oxford (1958).

27. S. Chandrasekhar, Radiative Transfer, Dover, New York (1960).

28. R. N. Thomas, Astrophys. J. 125, 260 (1957).

29. A. G. Hearn, Proc. phys. Soc. 84, Il (1963).

30. M. Dobrowolny and F. EngelmanN, Il Nuovo Cimento 37, 965 (1965).

31. A. UnSÖLd, Physik der Sternatmosphären, Springer Verlag (1955).

32. G. N. Walson, Theory of Bessel functions, Cambridge Univ. Press (1922).

33. W. de. Groot, Physica 9, 263 (1929); Z. Phys. 50, 559 (1928).

34. R. LaDenburg and S. LeVY, Z. Phys. 65, 189 (1930).

35. E. F. M. van der Held, Thesis, Utrecht 1932.

36. J. VAN. SUCHTELEN, private communication.

37. F. W. Hofmann and H. KoHn, J. opt. Soc. Amer. 47, 151 (1957).

38. W. Behmenburg, H. Kohn and M. MaILÄnder, JQSRT 4, 149, 163 (1964).

39. R. D. Present, Kinetic Theory of Gases, McGraw-Hill, New York (1958).

40. E. H. Kennard, Kinetic Theory of Gases, McGraw-Hill, New York (1938).

41. G. D. Chapman and L. Krause, Can. J. Phys. 43, 563 (1965).

42. C. van Trigt, Tu. Hollander and C. Th. J. Alkemade, JQSRT 5, 813 (1965).

43. A. B. Callear, Appl. Opt., Suppl. on Chem. Lasers, 145 (1965). 